\title{
Separation of Amylose and Amylopectin in Corn Starch Using Dual-programmed Flow Field-Flow Fractionation
}

\author{
Woon-Jung Kim, Chul Hun Eum, ${ }^{\dagger}$ Seung-Taik Lim, Jung-Ah Han, ${ }^{\sharp}$ Sang-Guan You, ${ }^{\sharp}$ and Seungho Lee ${ }^{*}$ \\ Department of Chemistry, Hamam University, Daejeon 305-811, Korea. "E-mail: slee@hmuackr \\ ${ }^{\dagger}$ Korea Institute of Geoscience and Mineral Resources, Daejeon 305-350, Korea \\ ${ }^{4}$ Department of Food Science and Technology, Korea University, Seoul 136-701, Korea \\ ${ }^{\S}$ Division of Human Environmental Sciences, Sangmyung University, Seonl 110-743, Korea \\ "Department of Marine Bioscience and Technology, Kangnung University, Kanghung 210-702, Korea \\ Received July 30,2007
}

Key Words : Corn starch, Amylose, Amylopectin. Flow field-flow fractionation (FFFF), Dual-programming

Starch, a major storage polysaccharide in plants, is used in a wide range of industries including confectionery, beverage or liquid flavor emulsions, pharmaceuticals, cosmetic products, inks, etc. ${ }^{1.2}$ Starch is a mixture of two macromolecular $\alpha$-glucans, linear amylose and branched amylopectin. ${ }^{3}$

Starch applications require dissolution (or dispersion) in aqueous media under non-degradation conditions. Functional properties of starch are influenced by the average molecular weights $(M)$ and molecular weight distributions (MWD) of the amylose and amylopectin, as was demonstrated in gels, ${ }^{+}$extrusion ${ }^{5}$ and pastes. ${ }^{6}$ Accurate determination of $M$ and MWD of the amylose and amylopectin requires separation of the two components. Both amylose and amylopectin are usually of very high molecular weights, and are difficult to separate.

Chromatography-related techniques have been widely used for starch analysis, including size-exclusion chromatography (SEC), ${ }^{7-12}$ high-performance anion-exchange chromatography (HPAEC). ${ }^{13-15}$ Often chromatographic analysis of starch is not satisfactory due to degradation and/or adsorption of the sample components during elution through packed columns. ${ }^{16-18}$ Recently, SEC coupled to multi-angle light scattering (MALS) has been demonstrated to be a useful tool for analysis of polymer systems, where SEC provides separation and MALS the molecular weight. ${ }^{19-23}$ SEC-MALS has been used for analysis of wheat amylopectin. ${ }^{17}$ The $M$ and the radius of gyration $\left(r_{g}\right)$ measured by SEC-MALS were significantly lower than those obtained by the batch mode of MALS due to degradation of the amylopectin during the passage through the packed column. ${ }^{23.24}$ It was also found that some of large amylopectin molecules are adsorbed. Based on the recovery test, a significant portion $(17.7-63.7 \%)$ of the sample was not recovered, resulting in reduction in measured $M^{25}$ It has been reported that, although the starch sample has been completely dissolved in water, only $86 \%$ of the sample has been recovered through SEC columns. ${ }^{16}$

Field-flow fractionation (FFF) is a family of techniques that are useful for separation of wide range macromolecules and colloidal particles. ${ }^{26.27}$ FFF possesses several merits over SEC for separation of ultrahigh molecular weight polymeric samples. Unlike in SEC, there is no packing material, minimizing degradation or adsorption of large molecules. Also there is no so-called "total exclusion limit" in FFF, and the applicable range in molecular weight is usually extended further to much higher molecular weights than in SEC. In addition, the fractionation range, resolution, and analysis time can be controlled rather easily by adjusting the flow rate and the field strength. Granular starch has been analyzed using sedimentation FFF. $^{28}$ Also derivatized potato amylopectins have been successfully analyzed by on-line coupling of flow FFF and multi angle light scattering (MALS) ${ }^{29}$ Yet there has not been reports on separation of amylose and amylopectin in aqueous solution of a starch by FFF. Among the members of FFF family, flow FFF (FIFFF) has been widely used for separation of water-soluble polymers and biological macromolecules. ${ }^{30-34}$ The aim of this work is to investigate the capability of FIFFF for separation of amylose and amylopectin in com starches.

In FIFFF, the retention time $t_{\mathrm{r}}$ of a component is related with its diffusion coefficient $D$ by ${ }^{30.35-38}$

$$
D=\frac{w^{2} V_{c}}{6 t_{r} V}
$$

, where $w$ is the channel thickness, $V_{\mathrm{c}}$ and $V$ are the flow rates of the cross-flow and the channel-flow, respectively. Using eqn (1), $D$ can be measured for each $t_{5}$ over the envelope of the elution curve. This relationship between $D$ and $t_{5}$ was recognized as an effective way for determining $D$ for proteins, viruses, and latex beads. ${ }^{38+1} D$ can be expressed as a function of molecular weight by ${ }^{+2}$

$$
D=\frac{R T}{6 \pi \eta N_{0}}\left(\frac{10 \pi N_{0}}{3 K}\right)^{1 / 3}\left(M_{v}\right)^{-(1+\alpha) / 3}
$$

, where $R$ is the universal gas constant, $N_{0}$ the Avogadro's number, $M$, the viscosity-average $M$, and $K$ (in $\mathrm{dL} / \mathrm{g}$ ) and $\alpha$ the Mark-Houwink constants. Retention time $t_{r}$ is also related with the hydrodynamic diameter, $d_{\mathrm{II}}$ by 30,38

$$
t_{r}=\frac{\pi w^{2} \eta V_{c}}{2 k T V} d_{H}
$$

, where $\eta$ is the viscosity of the carrier liquid, $k$ the 

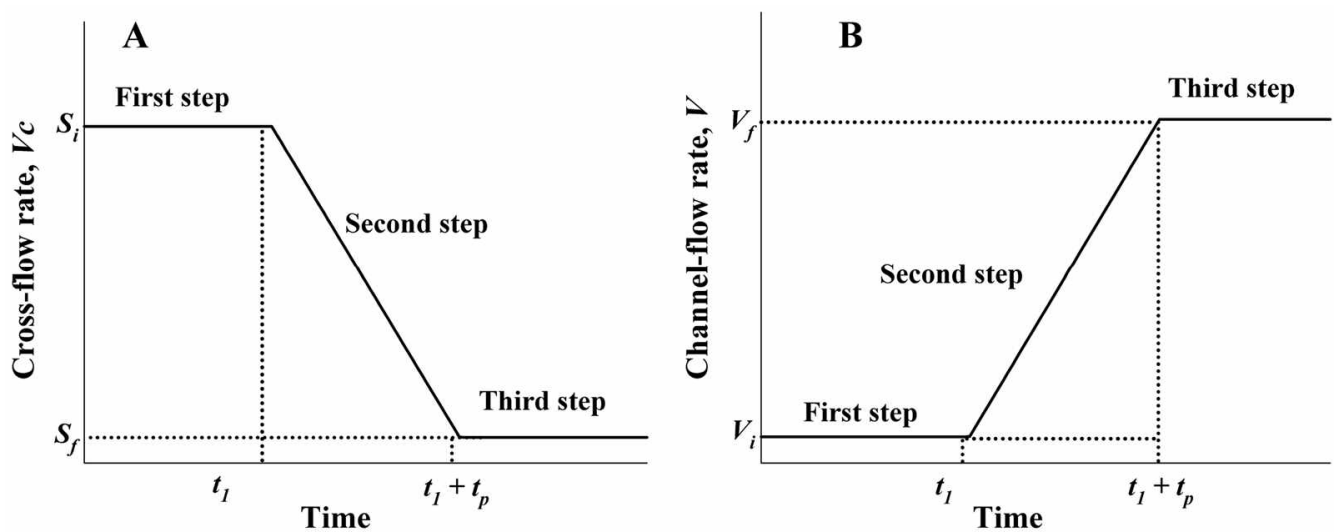

Figure 1. Profiles of cross-flow rate, $V_{s}(\Lambda)$ and channel-fiow rate, $V(B)$ in a linear dual-programıned FIFFF.

Boltzmann constant, $T$ the absolute temperature. It can be seen from $\mathrm{Eq}$. (1-3) that FIFFF provides separation of components based on either molecular weight $\left(M_{1}\right)$ or hydrodynamic diameter $\left(d_{11}\right)$.

In FFF, the field (and/or the flow rate)-programming can be used to adjust the resolution and the analysis time, where the field (and/or the flow rate) is varied during a run. Typical programmed FFF begins with a high level of field strength suitable for retention of the smallest components, and then the field strength is gradually reduced to allow elution of the components having larger diameters. ${ }^{+3,+4}$ The quantitative expressions applicable to non-programmed (isocratic) FFF can be extended to programmed FFF. In this study, a dualprogramming was used, where $V_{c}$ was reduced linearly while $V$ was increased linearly as shown in Figure I. A linear reduction of $V_{c}$ (Figure I-A) is expressed by

$$
V_{c}(t)=V_{c i}\left[\frac{t_{1}+t_{p}-t}{t_{p}}\right]
$$

over the time interval, $t_{1} \leq t \leq\left(t_{1}+t_{\mathrm{p}}\right)$. $V_{\mathrm{ci}}$ is the initial $V_{\mathrm{c}}, t_{1}$ the pre-decay time, the time duration of the first step, and $t_{\mathrm{p}}$ the duration of programming. Likewise, a linear increase of $V$ (Figure 1-B) can be expressed by

$$
V(t)=V_{i}\left[\frac{t_{1}+t_{p}-t}{t_{p}}\right]
$$

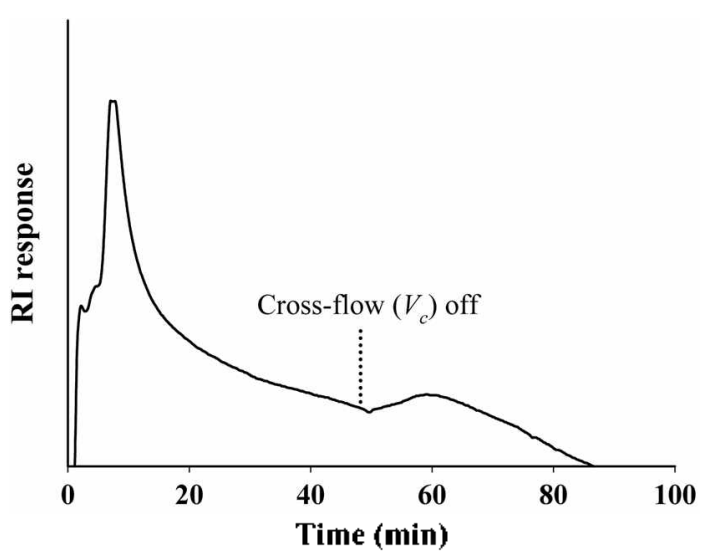

Figure 2. FIFFF fractogram of Nonnal corn starch obtained at $V_{\varepsilon}$ and $V$ of 0.2 and $0.25 \mathrm{~mL} / \mathrm{min}$, respectively.
Figure 2 shows a FIFFF fractogram of Normal com starch obtained at $V_{c}=0.2$ and $V=0.25 \mathrm{~mL} / \mathrm{min}$, respectively. The first peak (corresponding to amylose fraction) ${ }^{16-1822}$ is eluted at around $8 \mathrm{~min}$, after which the baseline keeps drifting down continuously. When $V_{\mathrm{c}}$ was tumed off at around 45 min, the remainder of the sample (mainly the amylopectir fraction) was eluted, indicating the amylopectin fraction is retained too much even under this mild (low $V_{c}$ ) condition.

To improve resolution, a dual-programming was employed. Figure 3 shows a FIFFF fractogram of Normal corn starch obtained by a linear dual-programming with $t_{1}=t_{p}=$ $20 \mathrm{~min}, V_{c i}=0.763, V_{c f}=0.058, V_{i}=0.214, V_{f}=0.919 \mathrm{~mL} /$ $\mathrm{min}$, respectively. The amylose (earlier eluting one) and the amylopectin (latter) fractions are well separated. The area ratio under the elution curves of amylose/amylopectin is $36 /$ 64. Accurate conversion from the area to concentration requires area/mass calibration and correction for the change in flow rate, etc. Accurate quantitative analysis was not possible due to the lack of pure standards of amylose and amylopectin. It is interesting, however, that the area ratio determined without such correction is somewhat close to the nominal amylose/amylopectin ratio of $30 / 70$.

Figure 4 shows a FIFFF fractogram of the High-Amylose com starch obtained at the same conditions as in Figure 3.

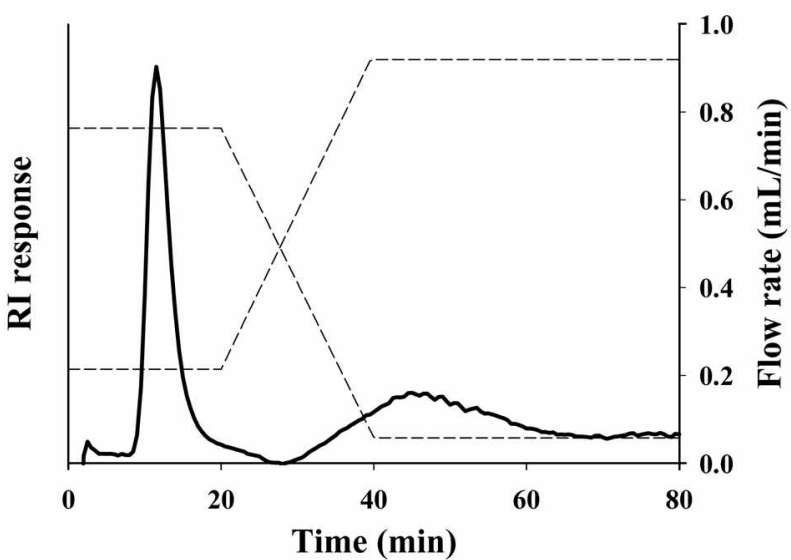

Figure 3. FIFFF fractogram of Nonnal com starch obtained by a linear dual-programming. Programming parameters are: $t_{t}=t_{p}=20$ $\min , V_{c t}=0.763, V_{c f}=0.058, V_{f}=0.214$, and $V_{f}=0.919 \mathrm{~mL} / \mathrm{min}$. 


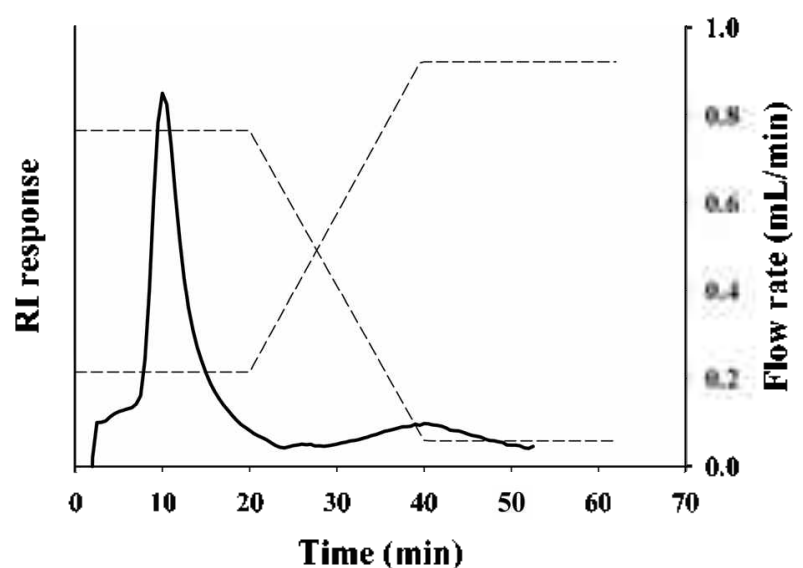

Figure 4. FIFFF fractograun of High- $\Lambda$ mylose corn starch obtained at the same condition as in Figure 3 .

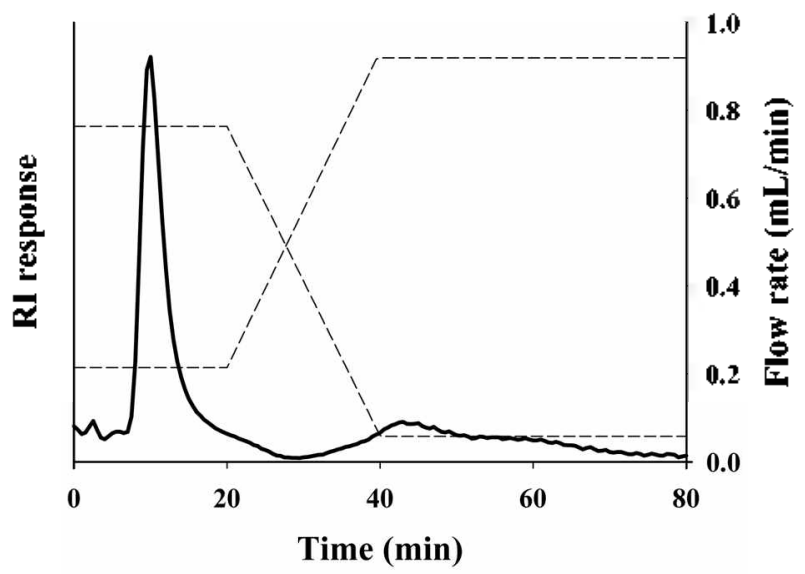

Figure 5. FIFFF fractogram of 7 days-old Normal corn starch solution obtained at the same condition as in Figure 3.

As in Figure 3, the two fractions are well separated. The area ratio of the elution curves of amylose/amylopectin is $75 / 25$, which is again somewhat close to the nominal ratio of $70 / 30$. As shown in both Figure 3 and 4 that the $M$ (or the size) distributions of the amylopectin fractions are much narrower than the amylopectin fractions in both Normal and HighAmylose com starches.

Figure 5 shows a FIFFF fractogram of the 7 days-old Normal con starch solution obtained at the same conditions as in Figure 3. The area ratio was changed from $36 / 64$ (Figure 3) to 52/48, which is probably due to degradation of the amylopectin fraction during storage. It seems the starch solutions prepared in this study is not quite stable, and need to be freshly prepared for each analysis.

Table 1 shows the average molecular weights $\left(M_{v}\right)$ and hydrodynamic diameters $\left(d_{\mathrm{H}}\right)$ of the Nonnal and the HighAmylose conn starches determined by Equations (2) and (3) respectively. As expected, $M_{\mathrm{V}}$ and $d_{\mathrm{H}}$ of the amylose fractions are much lower than those of the amylopectin fractions for both samples. It is likely that the $M$ and $d_{\mathrm{H}}$ values shown in Table I are smaller than the actual values due to the steric effect of these large molecules, which usually results in faster elution than predicted by FFF theory.
Table 1. Average molecular weights and sizes determıned by FIFFF for amylose and anylopectin fraction of corn starches

\begin{tabular}{lccc}
\hline Sample & $\begin{array}{c}\text { Molecular } \\
\text { weight" } \\
\left(\times 10^{6} \mathrm{Da}\right)\end{array}$ & $\begin{array}{c}\text { Hydrodynamic } \\
\text { diameter } \\
(\mathrm{nm})\end{array}$ \\
\hline Nomal & Amylose fraction & 0.144 & 13.4 \\
com starch & Amylopectin fraction & 43.2 & 460 \\
High-Amylose & Amylose fraction & 0.112 & 12.0 \\
com starch & Amylopectin fraction & 19.6 & 310 \\
\hline
\end{tabular}

Detemined by Equation (2) with Mark-J Iouwink coefficients, $K=1.32$ $\times 10^{-4} \mathrm{dL} / \mathrm{g}$ and $\alpha=0,68$. Determined by Equation (3)

In summary, FIFFF has been tested for separation of amylose and amylopectin in aqueous solution for the first time. A dual programmed FIFFF provided a good separation of the amylose and the amylopectin fractions of com starches. If further optimized, FIFFF can become a useful tool for analysis of starches and related materials. For accurate determination of molecular weight and the hydrodynamic sizes of the amylose and amylopectin fractions, online coupling of a multi angle light scattering (MALS) with FIFFF is suggested.

\section{Experimental Section}

Starch solution preparation. Two types of com starches (High-Amylose and Normal) were used in this study. The High-Amylose and Normal com starches have the amylose/ amylopectin ratio of $70 / 30$ and $30 / 70$, respectively. First, the starch sample $(10 \mathrm{mg})$ was wetted with $100 \mu \mathrm{L}$ ethanol and then $1 \mathrm{~mL}$ of $1 \mathrm{M} \mathrm{NaOH}$ was added. The mixture was heated for $2 \mathrm{~min}$ at $70^{\circ} \mathrm{C}$ in a water bath. Then $7.9 \mathrm{~mL}$ of the FFF carrier liquid (water containing $0.15 \mathrm{M} \mathrm{NaNO}_{3}$ ) was added, and the solution was neutralized by adding $1 \mathrm{~mL}$ of $\mathrm{I}$ $\mathrm{M} \mathrm{HCl}$. Finally the solution was autoclaved for $20 \mathrm{~min}$ at $121{ }^{\circ} \mathrm{C}$. The final concentrations of starches were about $0.1 \%$.

Flow field-flow fractionation (FIFFF). The accumulation wall of the FIFFF channel is an YM10 membrane (Amicon, Inc., USA) having $M$-cutoff of $10,000 \mathrm{Da}$. The Mylar spacer has dimensions of $29.3 \mathrm{~cm}$ in tip-to-tip length, $2.0 \mathrm{~cm}$ in breath, and $0.0194 \mathrm{~cm}$ in thickness. The channel volume was measured by the elution of the void peak to be $1.134 \mathrm{~mL}$. The FlFFF channel was positioned vertically to avoid the influence of the gravity. Sample solution was injected using a $20 \mu \mathrm{L}$-loop injector (Rheodyne model 7725 , Cotati, CA, USA), and eluting samples were monitored by a refractive index detector (Shodex RI-71, Showa Denko, Tokyo, Japan). Two microcomputer-controlled $6000 \mathrm{~A}$ pumps (Waters Corp., Milford, MA, USA) were used to provide $V_{c}$ and $V$. For dual-programming, the cross-flow pump was plumbed in a circulation mode, so that the $V_{c}$ coming out of the chanmel goes back to the inlet of the cross-flow pump.

Acknowledgements. This paper has been supported by the 2007 Hannam University Research Fund. SL acknow- 
ledges the support from KICOS for the Joint Research Program between Korea and Italy in 2007.

\section{References}

1. Patricia, L. B.; Frederick, G. M.; Robert. H. M. Int. J. Biological Macromol. 1999, 26, 193.

2. Pompilia, I. S.; Frandois, R.; Iman, H.; Michel, P.; Mircea, A. M. Carbohydrate Res. 2000, 323, 163.

3. French, D. Organization of Starch Gronules in Starch: Chemistry. and Technotog:; Whistler, R. L.; BeMiller, J. N.; Paschall, E. F., Eds.; Academic Press: Orlando, FL, 1984; p 183.

4. Clark, A. H.; Gidley, M. J.; Richardson, R. K.; Ross Murphy, S. B. Macromolectles 1989. 22.346.

5. Della Valle, G.; Colonna, P.; Patria, A.; Vergnes, B. J. Rheology 1996, 40, 347 .

6. Doublier, J. L.; Colonna, P.; Mercier, C. Cereal Chem. 1986, 63, 240.

7. Jane, J. L.; Chen, J. F. Cereal Chen. 1992, 69,60.

8. Takeda, Ch.; Takeda, Y; Hizukuri, S. Carbohydrate Res. 1993, 246,273 .

9. Shibanuma, K.; Takeda, Y.; Hizukuri, S.; Shibata, S. Carbohydrate Polyners 1994, 25, 111 .

10. Ong, M. H.; Blanshard, M. V. J. Cereal Sci. 1995, 21, 251 .

II. Striegel, A. M.; Timpa, J. D. Carbohydrate Res. 1995, 267, 271.

12. Charoenkul, N.; Uttapap, D.; Pathipanawat, W.; Takeda, Y. Carbohydrate Polymers 2006, 65, 102.

13. Shi, Y, C.; Seib, P. A. Carbohydrate Polyners 1995, 26, I4I,

14. Bello-Perez, L. A.; Paredes-Lopez, O.; Roger, P.; Colonna, P. Food Chem. 1996, 56, 171.

15. Hanashiro, I.; Abe, J. I.; Hizukuri, S. Carbohydrate Res. 1996 , $283,151$.

16. Bello-Perezt, L. A.; Roger, P.; Colonna, P. J. Cered Sci. 1998, 27. 267.

17. You, S.; Fiedorowicz, M.; Lim, S.-T. Cereal Chem. 1999, 76(1), 116.

18. Picton, L.; Bataille, I.; Muller, G. Carbohthrate Pohmters 2000. 42,23 .

19. Roger. P.; Colonna. P. Carbohudrate Polymers 1993, 21, 83.

20. Roger, P.; Colonna, P. Iht. J. Biological Macromol. 1996, 19, 51.
21. Wyatt, P. J. J. Anal. Chem. Acta 1993, 272, 1.

22. Millard, M. M.; Dintizis, F. R.; Willell, J. L.; Klavons, J. A. Cereal Chem. 1997, 74,687.

23. Hanselmann, R.; Ehrat, M.; Widmer, H. M. Slarch/Stacrke 1995 46,345 .

24. Aberle, Th.; Burchard, W.; Vorweg, W; Radosta, S. Starch Stacrke 1994, 46, 329.

25. Randall, R. C.; Phillips, G. O.; Williams, P. A. Food. Hydrocolloids $1989,3,65$.

26. Giddings, J. C.; Benincasa, M. A. J. Liq. Chronatogr 1992. $15(10), 1729$.

27. Kirkland, J.; Dilks, C. H.; Rementer, S. W. Anal. Chem. 1992, 64, 1295 .

28. Moon, M. H.; Giddings, J. C.J. Food Sci. 1993, $58, \mathrm{I} 166$.

29. Lee, S.; Nilsson, P.-O.; Nilsson. G. S.; Wahlund, K.-G. J. Chront. A $2003,1011,111$.

30. Giddings, J. C. Chem. Eng. News 1988, 66(O) 10), 34.

31. Giddings, J. C. J. Chem, Edu, 1973, 50, 667 .

32. Ji, E.; Choi, S.-H.; Yoon, K. R.; Chun, J.-H.; Lee, S. Bull. Kor Chem. Soc. 2006, 27(9), 1433.

33. Kang, D. Y.; Son, M.-S.; Eum, C. H.; Kim, W.-S.; Lee, S. Bull. Kor, Chem, Soc. 2007, 28(4),613.

34. Peterson, R. E.; Myers, M. N.; Giddings, J. C. Sep. Sci. Technol. $1984,19,307$

35. Koch, T.; Giddings, J. C. Anal. Chem, 1986, $58,994$.

36. Giddings, J. C.; Yang, F. J. F.; Myers, M. N. Anal. Chem. 1976, $48,1126$.

37. Giddings, J. C.; Yang. F. J. F.; Myers, M. N. Science 1976, 193 , 1244.

38. Giddings, J. C.; Yang, F. J. F.; Myers, M. N. J. Virol, 1977, 2I, 131.

39. Giddings, J. C.; Yang, F. J. F.; Myers, M. N. Anal. Biochem. 1977, 81,395 .

40. Yang, F. J. F.; Myers, M. N.; Giddings, J. C. Anal. Chent 1974 46. 1924 .

41. Roger, P.; Bello-Perez, L. A.; Colonna, P. Polymer 1999, $40,6897$.

42. Williams, P. S.; Giddings, J. C.; Beckett, R. J. Liq. Chromatogr: $1987,10,1961$.

43. Ratanathanawongs, S. K.; Giddings, J. C. Anal. Chem. 1992, 64. 6.

44. Moon. M. H.; Kang. D.; Hwang. I.; Williams. P. S. J. Chromatogr. A 2002, 955(2), 263. 